\title{
Aproximación al rol del profesor de educación básica primaria en Colombia'
}

\section{Approach to the role of the primary basic education teacher in Colombia}

José Eriberto Cifuentes Medina

Derly Francedy Poveda Pineda ${ }^{3}$

Universidad Pedagógica y Tecnológica de

Colombia

\section{RESUMEN}

En el proceso de elaborar una hermenéutica del rol del profesor de educación básica primaria, es vital la comprensión del sistema educativo del país; desconocer los fundamentos epistemológicos del ser del maestro es semejante a olvidar parte de la esencia y las

$1 \quad$ Artículo de Reflexión

2 Investigador Asociado (I) SNCTel, convocatoria 833. Magister en Educación, Especialista en Evaluación Educativa, Licenciado en Teología, Licenciado en Filosofía y Educación Religiosa, Universidad Santo Tomás. Especialista en Pedagogía y Docencia, Fundación Universitaria del Área Andina. Investigador Grupo de Investigación SIEK. Profesor de la Licenciatura en Educación Básica Primaria, Escuela de Ciencias Humanísticas y Educación, Facultad de Estudios a Distancia, Universidad Pedagógica y Tecnológica de Colombia. Orcid ID: https://orcid.org/0000-0001-5702620X E-mail: joseeriberto.cifuentes@uptc.edu.co

3 Magister en E-learning, Especialista en Gerencia Educacional, Licenciada en Ciencias de la Educación Informática Educativa. Universidad Pedagógica y Tecnológica de Colombia. Investigadora Grupo de Investigación SIEK. Profesora de la Licenciatura en Educación Básica Primaria. Escuela de Ciencias Humanísticas y Educación, Facultad de Estudios a Distancia, Universidad Pedagógica y Tecnológica de Colombia. Orcid ID: https://orcid.org/0000-0002-1616112X E-mail: derly.poveda@uptc.edu.co vivencias en cada comunidad educativa en donde es activa su presencia.

La investigación es de corte documental y de revisión de fuentes secundarias. Siendo objeto fundamental y eje dinamizador la descripción del rol del profesor de educación primaria en Colombia según la normatividad vigente, el papel de las escuelas normales, las universidades a través de las facultades de educación en su formación y la importancia de su presencia en las comunidades educativas en el ámbito rural y urbano; según las dinámicas de cada contexto y escenario entre otros elementos de consideración para el objeto de estudio.

Entre los aspectos que sobresalen en el rol del profesor de educación básica primaria se infiere que aún falta un estudio concluyente y categórico que conduzca a razonamientos denotativos y estudios comparados de los avances y retrocesos de este nivel educativo en Colombia tanto en la formación de profesores 
como en la educación impartida por ellos a los niños. El objetivo de la investigación es orden teórico, por lo que se hace necesario derivar otras investigaciones en detalle del rol del profesor de Educación Básica Primaria en el ambiente rural y urbano, promoviendo el análisis de los modelos pedagógicos, las prácticas pedagógicas, los decretos de escalafón y las directrices emanadas del Ministerio de Educación Nacional.

\section{PALABRAS CLAVE:}

Profesor, Educación Básica Primaria, Sistema Educativo, Colombia

\section{ABSTRACT}

In the process of developing a hermetic role of primary school teacher, understanding of the country's educational system is vital; to ignore the epistemological foundations of being a teacher is similar to forgetting part of the essence and the experiences in each educational community where his presence is active.

The research is of documentary cut and revision of secondary sources. Being a fundamental object and driving force is the description of the role of primary education teacher in Colombia according to the current regulations, the role of normal schools, universities through the education faculties in their formation and the importance of their presence in the communities Education in rural and urban areas; According to the dynamics of each context and scenario among other elements of consideration for the object of study.

Among the aspects that stand out in the role of the elementary primary school teacher, it is inferred that a conclusive and categorical study of denotative reasoning and comparative studies of the advances and setbacks of this level of education in Colombia is still lacking both in teacher training and in the education given by them to the children. We consider the present study of a broad research, so it is necessary to derive other researches in detail of the role of the teacher of primary education in rural, urban and urban environment among others, promoting in analysis of pedagogical models, pedagogical practices, Decrees of ladder and the directives emanated of the Ministry of National Education.

KEYWORDS: Teacher, Education Basic Primary, Educational System, Colombia.

\section{INTRODUCCIÓN}

La ilación del artículo hace un recorrido por tres categorías: marco normativo del sistema educativo colombiano, las instituciones formadoras de formadores y el papel del profesor de básica primaria. Desde la normatividad vigente que regula el sistema educativo colombiano, a través de la visibilización de la Ley 30 de 1992 y la Ley 115 de 1994: ley general de educación) y los decretos de regulación necesarios para cada caso, es decir, que la ocupación que los estudios referidos se constituyen en soporte teórico para consolidar el sistema educativo colombiano en los últimos 25 años posteriores a la Constitución de 1991.

El estudio en perspectiva normativa en Colombia contribuye a la comprensión del rol del profesor de educación básica que ahora es objeto de estudio, sin embargo, se ocupa también de manera sucinta de las instituciones que forman los profesores de este nivel de educación: Escuelas Normales y Universidades, al igual que manera relativa se hace alusión a su esencia vital en el desarrollo de las comunidades en donde se hace visible su presencia por su liderazgo y no solo trasmisión de contenidos sino construcción de conocimiento en el proceso de enseñanza y aprendizaje en los cinco grados que comprenden la Educación Básica Primaria. Es relevante la investigación en la medida que al desconocer el rol esencial del profesor de básica se puede perder la identidad de una parte de la comunidad educativa. 
Para investigar el rol del profesor de educación básica primaria el Colombia es importante en el desarrollo hermenéutico y epistemológico no solo desde la norma que regula su formación, vinculación, profesionalización, capacitación y asignación salarial, sino que es necesario también su desarrollo profesional que en su vocación desempeña en el proceso de enseñanza y la huella que deja en cada uno de sus estudiantes. El profesor de educación básica primaria, se constituye en una piedra angular del desarrollo integral de los educandos que hacen parte de este nivel educativo, es uno de los sujetos de la comunidad educativa y que desde luego a de contribuir a la construcción de una sociedad digna en medio de la diversidad para el progreso y desarrollo social de cada comunidad.

\section{CONSIDERACIONES TEÓRICAS}

El hilo conductor de la investigación hace un esbozo por la normatividad en relación a la educación: constitución de 1991, Ley 30 de 1992, Ley 115 y se hace mención de algunos de los decretos reglamentarios cuando es necesario y es parte del objeto de estudio. Las categorías de análisis contribuyen a la ubicación normativa del rol del profesor de educación básica primaria, avanzando por las instituciones que hacen parte de su formación, profesionalización y capacitación dando paso a su campo de acción en los diferentes escenarios: urbano y/o rural y según los mismos modelos y practicas pedagógicas. El apoyo de diversos autores son elementos de base primordial para abordar el rol del profesor de educación básica primaria en Colombia como parte del sistema educativo.

\section{GENERALIDADES DE NORMATIVIDAD DEL SISTEMA EDUCATIVO EN COLOMBIA}

En el propósito de escudriñar el rol del profesor de educación básica primaria, se acude a la norma vigente en punto de partida se evidencia la Constitución Política de 1991, Ley 30 de 1992,
Ley 115 de 1994 y decretos reglamentarios. Desde la norma se puede entrelazar las funciones, el perfil y el campo de acción del profesor y desde allí se puede configurar su rol, al menos en la ley que conlleva a la estandarización y puede distar de la realidad en el ejercicio profesional de la enseñanza en cada contexto particular de la geografía nacional.

La Constitución de 1991 y los principios de la educación. En Colombia, a partir de la Constitución de 1991, durante el gobierno de Cesar Gaviria (1990-1994) se inició una época de reformismo educativo. El 4 de julio de 1991 fue proclamada una nueva Constitución Nacional de Colombia, que suspendía a la de 1886. En este sentido Quiceno, Sáenz y Vahos (2004) sostienen que:

Esta Constitución recogió aspiraciones políticas y sociales acumuladas durante décadas y fue la culminación de un pacto de paz y convivencia establecido por el Estado con varios grupos guerrilleros que se habían incorporado a la vida civil, constituyéndose en una carta garantista en relación con los derechos fundamentales de los ciudadanos y las minorías étnicas.

La nueva Constitución de Colombia en los artículos 67, 68, 69 y 70, estableció los principios en el campo de la educación. 
Tabla $N^{\circ} 1$

Principios de la educación propuestos por la Constitución de 1991

\begin{tabular}{cl}
\hline PRINCIPIO & DESCRIPCIÓN \\
\hline $\mathbf{1}$ & La Educación como un derecho Fundamental \\
\hline $\mathbf{2}$ & La prevalencia de los derechos de los niños sobre los de otros sectores de la población \\
\hline $\mathbf{3}$ & La corresponsabilidad del Estado, la sociedad y la familia en la Educación \\
\hline $\mathbf{4}$ & La obligatoriedad de la educación básica hasta grado nueves grados \\
\hline $\mathbf{5}$ & La autonomía de las Universidades \\
\hline $\mathbf{6}$ & El derecho a la libre personalidad \\
\hline $\mathbf{8}$ & La educación bilingüe en las comunidades indígenas \\
\hline $\mathbf{9}$ & La libertad de enseñanza, investigación y catedra \\
\hline $\mathbf{1 0}$ & La obligatoriedad de la enseñanza de la educación cívica y de la Constitución \\
\hline
\end{tabular}

Fuente: Constitución de 1991

Al respecto, Ferro, Prieto \& Quijano (2009) sostienen que, la reforma educativa colombiana en la década de los noventa es consecuencia de las políticas mundiales de la economía de mercado, establecida por organismos multilaterales en relación con el fenómeno de globalización. Es así como el discurso económico se consolida bajo un esquema de fortalecimiento democrático y de igualdad social, impulsando estrategias de poder y de saber centradas en una serie de condiciones operativas e instrumentales, con el objetivo de concebir la institución educativa como una empresa. A partir de 1986, el Estado optó por una estrategia de descentralización en lo administrativo y financiero, para romper la situación de concentración casi total en el nivel central, lograda después de casi un siglo de esfuerzos centralizadores. Al respecto Tedesco (2000) afirma que:

"la descentralización y la mayor autonomía a las instituciones escolares ha sido históricamente reivindicada por solo educadores y los movimientos pedagógicos orientados a lograr mayor nivel de libertad de los actores del proceso educativo para innovar, para construir opciones curriculares y para adecuarse a la diversidad social y cultural de los alumnos".

Esta perspectiva pedagógica fue perdiendo importancia y en la década de los 90 , la descentralización fue promovida desde una configuración administrativa en donde los objetivos fundamentales fueron la necesidad de reducir el gasto público, su utilización más eficiente.

La 30 de 1992 y la educación superior en Colombia. La ley 30 hace parte de las reformas que se iniciaron a partir de la Constitución Política de Colombia de 1991. Se constituye en la Ley que "organiza el servicio público de la Educación Superior", y desde la autonomía universitaria consagrada en la Constitución Política de Colombia, reconoce a las Universidades varias facultades y funciones entre ellas "seleccionar a sus profesores, admitir a sus alumnos y adoptar sus correspondientes regímenes y establecer, arbitrar y aplicar sus recursos para el cumplimiento de su misión social y de su función institucional" (Ley 30).

Para el objeto de estudio refiere que las Universidades son autónomas en la selección de profesores que contribuirán a la formación 
de los profesores de básica primaria y también en la admisión de los estudiantes que aspiran a cursar esta carrera en el nivel de pregrado en el programa de: Licenciatura en Educación Básica Primaria o con nombres similares al ejercicio.

La Ley 30, no refiere directamente cual es el rol del profesor de básica primaria sino del profesor universitario en todo aquello que le infiere y concierne de perfil, dedicación, funciones, participación entre otros aspectos no objeto de presente estudio. Sin embargo, no se puede excluir su asistencia en la discusión dado que son los profesores universitarios en su rol quienes contribuyen a la profesionalización de los estudiantes egresados de las Escuelas Normales Superiores o directamente en la formación de quienes inician la formación pedagógica sin nociones de pedagogía, al igual que son participes de la capacitación de profesores de primaria

La Ley 115 de 1994: Ley General de la Educación. La Ley 115 señala las normas generales para regular el Servicio Público de la Educación y se apoya en el Decreto Reglamentario 1860 del mismo año. En la formación de profesores, las reformas educativas en la década de los noventa, parte de la Constitución Política de Colombia en su Capítulo II, según lo establecido en los Artículos 67 al 70 los cuales definen que "La educación es un derecho de la persona y un servicio público que tiene una función social que se garantice la autonomía universitaria en la igualdad de oportunidades". Y de la Ley 115 de 1994, en el Artículo 109 se encuentra estipulados los siguientes fines:
"Formar un Educador de la más alta calidad científica y ética, b) desarrollar la teoría y práctica pedagógica como parte fundamental del saber del educador, c) fortalecer la investigación en el campo pedagógico y el saber específico y d) preparar Educadores a nivel de pregrado y de postgrado para los diferentes niveles y formas de prestación del servicio educativo" (M.E.N., 1994).

La Ley 115, enmarca de manera especial el objeto de estudio que ahora se analiza en razón señala directamente al profesor de básica primaria e indica en varios de sus artículos de manera directa la relación entre el profesor y este nivel de educación, por ello será uno de las columnas de sostenimiento normativo de la presente disertación de carácter reflexivo.

En la Ley general de educación refiere los niveles de educación de Preescolar, Básica primaria, Básica secundaria y media en 12 grados. Se esquematiza los niveles en la tabla $\mathrm{N}^{\circ} 2$, pero el propósito es examinar de manera sucinta el rol del profesor en educación básica primaria para los 5 grados que se enmarca su campo de acción. 
Tabla $\mathrm{N}^{\circ} 2$

Niveles de Educación 12 grados

\begin{tabular}{|c|c|}
\hline NIVEL & DEFINICIÓN \\
\hline $\begin{array}{l}\text { PREESCOLAR } \\
\text { Un grado } \\
\text { (Artículo } 15 \text { al 18) }\end{array}$ & $\begin{array}{l}\text { La educación preescolar corresponde a la ofrecida al niño para su desarrollo } \\
\text { integral en los aspectos biológico, cognoscitivo, sicomotriz, socio-afectivo y } \\
\text { espiritual, a través de experiencias de socialización pedagógicas y recreativas. } \\
\text { Grado obligatorio. El nivel de educación preescolar comprende, como mínimo, } \\
\text { un (1) grado obligatorio en los establecimientos educativos estatales para niños } \\
\text { menores de seis (6) años de edad. }\end{array}$ \\
\hline $\begin{array}{c}\text { BÁSICA } \\
9 \text { grados } \\
\text { (Artículo } 19 \text { a 26) }\end{array}$ & $\begin{array}{l}\text { La educación básica obligatoria corresponde a la identificada en el artículo } 356 \text { de } \\
\text { la Constitución Política como educación primaria y secundaria; comprende nueve } \\
\text { (9) grados y se estructurará en torno a un currículo común, conformado por las } \\
\text { áreas fundamentales del conocimiento y de la actividad humana }\end{array}$ \\
\hline BASICA PRIMARIA & $\begin{array}{l}\text { Objetivos específicos de la educación básica en el ciclo de primaria. Los cinco } \\
\text { (5) primeros grados de la educación básica que constituyen el ciclo de primaria, } \\
\text { tendrán como objetivos específicos los siguientes: }\end{array}$ \\
\hline $\begin{array}{c}\text { (Artículo } 21,23 \text { a } \\
26)\end{array}$ & $\begin{array}{l}\text { a) La formación de los valores fundamentales para la convivencia en una sociedad } \\
\text { democrática, participativa y pluralista; b) El fomento del deseo de saber, de la } \\
\text { iniciativa personal frente al conocimiento y frente a la realidad social, así como } \\
\text { del espíritu crítico; entre otros }\end{array}$ \\
\hline $\begin{array}{c}\text { BASICA } \\
\text { SECUNDARI }\end{array}$ & $\begin{array}{l}\text { Objetivos específicos de la educación básica en el ciclo de secundaria. Los cuatro } \\
\text { (4) grados subsiguientes de la educación básica que constituyen el ciclo de } \\
\text { secundaria, tendrán como objetivos específicos los siguientes: a) El desarrollo } \\
\text { de la capacidad para comprender textos y expresar correctamente mensajes } \\
\text { complejos, orales y escritos en lengua castellana, así como para entender, } \\
\text { mediante un estudio sistemático, los diferentes elementos constitutivos de la } \\
\text { lengua; entre otros. }\end{array}$ \\
\hline
\end{tabular}

(Artículo 22 a 26)

La educación media constituye la culminación, consolidación y avance en el logro de los niveles anteriores y comprende dos grados, el décimo $\left(10^{\circ}\right)$ y el undécimo

MEDIA VOCACIONAL

(Artículo 27 a 35) $\left(11^{\circ}\right)$. Tiene como fin la comprensión de las ideas y los valores universales y la preparación para el ingreso del educando a la educación superior y al trabajo. La educación media tendrá el carácter de académica o técnica.

Fuente: Información recabada de la Ley 115 
En el campo de acción del profesor de básica primaria se evidencia de manera directa en artículo 21 y 23 , sin descuidar los artículos 19 , 20, 23 a 26 pero no está implicado en el 22 ya que corresponde a básica secundaria. El N²1 señala que son 5 grados de básica primaria y los objetivos de la misma al igual que el en 23 que señala que:

"Paraellogro delosobjetivos de laeducación básica se establecen áreas obligatorias $y$ fundamentales del conocimiento y de la formación que necesariamente se tendrán que ofrecer de acuerdo con el currículo y el Proyecto Educativo Institucional. Las áreas obligatorias y fundamentales que comprenderán un mínimo del $80 \%$ del plan de estudios, son los siguientes: 1. Ciencias naturales y educación ambiental, 2. Ciencias sociales, historia, geografía, constitución política y democracia, 3. Educación artística, 4. Educación ética y en valores humanos, 5. Educación física, recreación y deportes, 6. Educación religiosa, 7. Humanidades, lengua castellana e idiomas extranjeros, 8. Matemáticas y 9. Tecnología e informática" (Ley 115, Articulo 23)

Se expresa desde el marco jurídico-normativo el campo de acción del profesor de básica primaria que corresponde a 5 grados y de las asignaturas obligatorias y fundamentales que son: 9 en las cuales se ha de formar y que ha de enseñar a sus estudiantes. Se aclara que es relativo en la medida que depende su escenario de trabajo urbano y/o rural entre otras variables como escuela unitaria, educación para adultos teniendo en cuenta la educación para grupos étnicos, población campesina y rural, con las particularidades.

El rol del profesor de educación básica primaria, desde los principios que señala la Constitución Política de Colombia, la profesionalización, formación y capacitación de los docentes universitarios a los profesores objeto de estudio y lo que señala la ley general de educación; queda enmarcado el campo de acción de básica primaria. También otros artículos de la misma ley refieren al objeto de estudio al igual que leyes y decretos en relación a concursos de vinculación, profesionalización, capacitación e incluso de adición de asignaturas al plan de estudios que ha de enseñar.

Normatividad en relación a las licenciaturas en Colombia. Según Bohórquez (2017) entre los requisitos para ser profesor de básica primaria indica que ha de remitir al artículo 198 de la Ley 115 de 1994 y así mismo el artículo 68 del Decreto 1278 del 2002. Desde el Ministerio de Educación Nacional se pretende dar cumplimiento a la normatividad vigente $y$ para ello se ha visibilizado la exigencia a las instituciones formadoras de formadores en aras de la consecución de la calidad en la educación tanto a las Escuelas Normales Superiores como a los programas de licenciatura que ofrecen las Universidades.

Es necesario destacar también el decreto 2450 de 2015 por el cual se reglamentan las condiciones de calidad para el otorgamiento y renovación del registro calificado de los programas académicos de licenciatura y los enfocados a la educación, y se adiciona el Decreto 1075 de 2015, Único Reglamentario del Sector Educación. De igual manera, para el diseño del programa se rige por la resolución 2041 del 2016 Por la cual se establecen las características específicas de calidad de los programas de Licenciatura para la obtención, renovación o modificación del registro calificado. Por último, se fundamenta en el Programa Nacional de Formación de Educadores El Ministerio de Educación Nacional -MEN para desarrollar la política de calidad para el cuatrienio 2010 -2014 "Educación de Calidad, el Camino para la Prosperidad". Además, el Decreto 2566 de 2003, en el que se incorporan algunos esbozos de marco regulatorio, que se 
concretaron en la Resolución de características específicas No. 2755 de 2006, como también en las disposiciones en el Decreto 1295 de 2010, por el cual se "involucran conceptos que modifican la esencia de la educación a distancia, tales como clasificar la modalidad en dos, modalidad de educación a distancia".

Para el caso de atender grupos étnicos, se contemplará lo establecido en el Artículo 57 de la Ley 115 de 1994, que señala: "Lengua materna, en sus respectivos territorios, la enseñanza de los grupos étnicos con tradición lingüística, propia será bilingüe, en donde la lengua materna es la aborigen y la segunda lengua el español sin detrimento de lo dispuesto en el literal c) del Artículo 21 de la presente Ley". Asimismo, la Ley 13812010 que especifica el reconocimiento y preservación de las lenguas de los grupos étnicos existentes en Colombia. "como patrimonio inmaterial de Colombia y por tanto es necesario salvaguardar toda una tradición de pueblos nativos"

El entorno legal y normativo se constituye en elemento vital para el objeto de estudio en curso en razón la ubicación del profesor de educación básica primaria en Colombia desde la Constitución Política de 1991 y que también se evidencia las diversas reformas de la vinculación, profesionalización y capacitación del profesor para la efectividad y calidad en la enseñanza, buscando la cobertura de la educación y la reducción del analfabetismo en el país. Las reformas también se han dado en los contenidos de enseñanza, los cambios al currículo, la adición de asignaturas obligatorias, las diversas cátedras e incluso se ha introducido la necesidad de responder a las pruebas nacionales e internacionales estandarizadas, entre otros y diversos aspectos de relación a la educación básica primaria en Colombia.

\section{INSTITUCIONES EDUCATIVAS FORMADORAS DE PROFESORES}

En el marco de la segunda categoría de análisis: Instituciones Educativas formadoras de los profesores de básica primaria en Colombia, se señala de forma sucinta a las Escuelas Normales Superiores y las Universidades como espacios de formación, profesionalización, capacitación de los profesores que han de enseñar en este nivel de educación.

De las Escuelas Normales Superiores y la formación de profesores de Primaria. Entre las instituciones educativas formadoras de los profesores de básica primaria en Colombia, son las Escuelas Normales Superiores que la Ley 115 indica que la responsabilidad de la formación y actualización de los Educadores, del nivel de pregrado y postgrado, en las Universidades e instituciones de Educación Superior, en su Artículo 112 (M.E.N., 1994) y establece, en el mismo Artículo en su Parágrafo, que "las Escuelas Normales reformadas, con dos años más de educación después del grado once, podrán formar Educadores para el nivel Preescolar y el ciclo de Educación Básica Primaria" De igual manera en 1994, el Decreto 2903, con fundamento en la Ley 115 , contiene los procedimientos para la reestructuración de estas Escuelas Normales, siempre y cuando cuenten con la asesoría de una Universidad.

Las Escuelas Normales Superiores, a lo largo de la historia del profesor de básica primaria y de su formación, han tenido un papel relevante a nivel nacional y regional como;

"garantes de preservar y
fortalecer una cultura pedagógica a
través de la preparación de los maestros
en función de las necesidades del
país. Desde su creación en 1821, por
autorización del General Francisco de
Paula Santander, han surtido diferentes
procesos de transformación en la línea


de las dinámicas del sector educativo en nuestro país y las tendencias pedagógicas a nivel mundial sobre la formación de docentes; las ENS se constituyen así en instituciones destacadas por ser instituciones formadoras de maestros para la educación preescolar y básica primaria, dos niveles educativos claves en tanto que es en ellos donde se cimientan las bases para los futuros aprendizajes $y$ para la vida" (MEN).

Colombia cuenta con 32 departamentos y un total de 137 Escuelas Normales Superiores Acreditadas para la formación de profesores, para un promedio aproximado de 4,5 aproximado por departamento. Para el caso del departamento de Boyacá, esté posee 10 Instituciones Educativas con este carácter, 8 adscritas a la Secretaria de Educación del Departamento y 2 a la Secretaria de Educación de Tunja.
Se constituye las137 Escuelas Normales Superiores acreditadas de Colombia en instituciones que a la luz de la normatividad y de las exigencias del Ministerio de Educación Nacional, formadoras de profesores de básica primaria de alta calidad para enseñar las asignaturas que señala el artículo 23 de la Ley 115 y por ende han de contribuir en la formación de los niños que le son confiados por la sociedad y con el desarrollo de competencias y funciones, ser profesores lideres e impulsadores de la calidad de vida de la comunidad en donde desempeña su labor pedagógica.

Las Universidades y la profesionalización de Profesores de Primaria. En Colombia, la formación de profesores se reglamentó a partir de la expedición de la Ley 115 de 1994, la Ley 30 de 1992 y el Decreto 709 de 1996; con el propósito de mejorar la calidad de la formación de los profesores de todos los niveles y desde luego de básica primaria, para ello se ha encargo a las Instituciones Educativas según se describe en la Tabla $\mathrm{N}^{\circ} 3$.

Tabla $\mathrm{N}^{\circ} 3$

Formación de profesores

\begin{tabular}{|c|c|}
\hline I.E.S. & DESCRIPCIÓN \\
\hline $\begin{array}{c}\text { Escuelas Normales } \\
\text { Superiores }\end{array}$ & $\begin{array}{l}\text { La primera hace referencia a la formación de educadores en las Escuelas Normales } \\
\text { Superiores con el denominado Ciclo de Formación Complementaria, o grados } \\
\text { doce y trece, para la atención de la educación preescolar y básica primaria, cuya } \\
\text { profundización debe atender las necesidades específicas de las comunidades } \\
\text { adyacentes y estar articulada con una facultad de Educación de una Institución de } \\
\text { Educación Superior (IES) reconocida por el Ministerio de Educación Nacional. }\end{array}$ \\
\hline $\begin{array}{c}\text { Universidades } \\
\text { con Facultades de } \\
\text { Educación }\end{array}$ & $\begin{array}{l}\text { La segunda designa que las IES que tengan facultades de educación o unidad } \\
\text { académica dedicada a la educación, podrán formar licenciados en las diferentes } \\
\text { áreas del conocimiento consagradas en el artículo } 23 \text { de la Ley } 115 \text {, el preescolar, } \\
\text { necesidades educativas especiales y en Etnoeducación; pero, de igual manera, } \\
\text { podrán ofertar programas de formación de posgrados y continua, por medio de } \\
\text { ciclos de actualización, capacitación y entrenamiento; diplomados, cursos, cátedras, } \\
\text { programas de formación permanente de docentes (PFPD), entre otros, estos últimos } \\
\text { para la obtención de créditos que permitan el ingreso y ascenso de los docentes del } \\
\text { Decreto } 2277 \text { de } 1979 \text {. }\end{array}$ \\
\hline
\end{tabular}


Fuente: Información recabada de Díaz (2015)

Con este panorama y "desde esta perspectiva, los aspirantes y docentes en nuestro país pueden optar por diferentes procesos de formación, para ingreso, ascenso, cualificación y mejoramiento de su actividad académica y profesional (Díaz, 2015). Es posible y viable la formación de profesores de básica primaria de alta calidad desde las Escuelas Normales Superiores y también de las Universidades que a través de las Facultades de Educación contribuyen la profesionalización y preparación de los profesores que enseñan en el nivel de educación objeto de estudio de la presente disertación.

La Ley 30 de 1992, plantea dentro de los principios de la educación superior, "la formación integral, la cual debe traducirse en la preparación de profesionales no solo instruidos, especializados y competentes, sino que sean personas integrales con una alta calidad humana y comprometidos con el proceso de construcción reconstrucción nacional" (Morales, 1999). Apropiado para la formación de profesores de básica primaria, competentes en la enseñanza de las reas obligatorias y fundamentales, pero también seres humanos entre los humanos.

Las Universidades públicas y privadas con vocación originaria o en el tiempo, de formación de profesores de los diferentes niveles de educación cuentan con el reto de brindad una formación de alta calidad, tal como es exigencia para hoy para las universidades acreditación de alta calidad, también para los programas que oferta cada universidad y para el caso de las licenciaturas se señala en el decreto 2450 de 2015 y en la Resolución 18583 de 2017 y demás normatividad vigente como el Decreto 1330 de 2019 que de forma explícita, propenden por la calidad del programa de Licenciatura en Educación Básica Primaria pero también de los futuros profesionales en Educación Básica Primaria han de ser idóneos en su formación y desempeño profesional idóneo desde la misma formación en los resultados del aprendizaje coherentes con la realidad en contexto.

\section{EL PROFESOR DE EDUCACIÓN BÁSICA PRIMARIA}

El ser humano tiene en su esencia un vo-care, es decir, un llamado a cumplir una misión con su vida en el mundo. Algunos cuentan con la semilla en sí mismo de ser servidores de los demás a través de algo que es propio solo para los humanos y es el educare; conducir a sus semejantes por el camino del conocimiento. Ellos son los profesores, también conocidos como docentes o maestros, reconocidos como profesores de una disciplina y con la convicción de guiar a niños, adolescentes, jóvenes y adultos a descubrirse a sí mismo, a aprender unas competencias, construir conocimiento, manifestar la realidad, ver la vida de otra forma, forjar un futuro mejor y a defenderse en la vida.

Hombres y mujeres emprenden el cumplimiento de su misión como profesores, se inician desde la formación impartida en las Escuelas Normales Superiores, pasando por las Facultades de Educación en la formación pedagógica y didáctica propia para el ejercicio de la enseñanza. Según Duque Linares en sus obras: "El arte de ser Maestro" y "La misión de educar", a la luz de la realidad se hace una reflexión sobre el educar como un arte, en el acto educativo y pedagógico y en el proceso de enseñanza y aprendizaje, entre el maestro y el estudiante. 
En el ejercicio de la docencia especialmente en licenciados se ha de notar el compromiso por la tarea que le ha sido encomendada, por ello ha der ser feliz, pero ¿Cómo se sabe si es o no feliz?, afirma Duque que; "Cuando un maestro es feliz, es porque le gusta la docencia, lleva la pedagogía en la sangre, ve a sus alumnos como a sus hijos, él asume su papel de padre, los quiere y se preocupa por su futuro" (Duque, 2001:30). La coherencia del maestro con su quehacer es un arte, por ello educar es algo más que un trabajo, más que enseñar una disciplina; es en verdad una entrega total por formación integral y la búsqueda de la perfección de sus estudiantes en pro de una vida digna.

Por ello ha de tener funciones como: aporte a la cultura personal, búsqueda y asimilación de la cultura, regulación en el proceso del aprender, estimulación en la actividad del educando y evaluación de lo aprendido; así, el maestro es el verdadero profesional de la educación, con el apoyo de la filosofía, se ha de convertir en un gran maestro de la vida, que por misión ha de contribuir con la perfectibilidad de sus congéneres. Por lo tanto, el rol o papel del profesor de básica primaria en Colombia, es esencial para el desarrollo cognitivo de los niños y niñas que inician su periodo escolar posterior al grado obligatorio en Preescolar. Por ello, en los cinco grados que comprende la básica primaria son eje fundamental para el desarrollo integral de los estudiantes por los procesos de desarrollo del niño y la niña.

Además de los profesores y profesoras que desempeñan su labor profesional en Educación Básica Primaria, como afirma el Periódico al Tablero "es importante que los padres y madres de familia y la sociedad en general se sumen a este proyecto educativo para que acompañen a los niños, niñas y jóvenes por los caminos del conocimiento" y la formación en las diferentes dimensiones de la vida humana y social para logar un impacto coherente entre la enseñanza y aprendizaje para la vida. El profesor de básica primaria ha de estar preparado y formado para enseñar los contenidos de las áreas obligatorias y fundamentales que señala la ley:

“1. Ciencias naturales y educación ambiental, 2. Ciencias sociales, historia, geografía, constitución política y democracia, 3. Educación artística, 4. Educación ética y en valores humanos, 5. Educación física, recreación y deportes, 6. Educación religiosa, 7. Humanidades, lengua castellana e idiomas extranjeros, 8. Matemáticas, 9. Tecnología e informática" (Ley 115, Articulo 23)

Y las demás áreas que con las reformas de los últimos años se han hecho visibles y obligatorias como el caso de: Catedra de la Paz, según la Ley 1732 de 2014 y el Decreto 1030 de 2015. Su tarea es fomentar la felicidad de los niños de básica primaria que tiene a cargo, a través de la enseñanza de los contenidos, pero también de las experiencias, promoviendo a través de los estilos de enseñanza, pero también de los estilos de aprendizaje la construcción conceptual y también de la vida como una posibilidad para entender la calidad de vida de un sujeto, pero también de la participación en la comunidad de manera social.

Principales dimensiones del profesor de básica primaria. El profesor que enseña en básica primaria ha de ser como indica Lozano (2011) un verdadero y auténtico profesor ha de ser prudente y sabio, en el ejercicio de su labor, ha de estar asistido por cualidades de persona integral y le es necesario desarrollar en equilibrio al menos doce dimensiones, para brindar un servicio de calidad a sus estudiantes. 
Figura 1

Dimensiones de un docente, Según Lozano (2011).

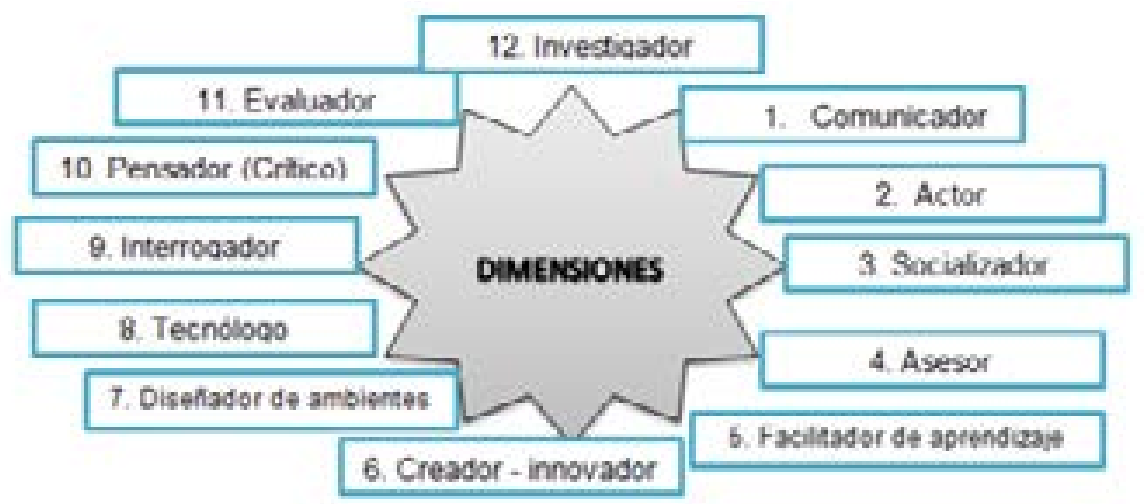

Fuente: Los autores

Los docentes del siglo XXI, que desempeñan su labor en básica primaria, requieren de una preparación idónea y de cualificación permanente acordes a la vanguardia de los avances tecnológicos y de los cambios emergentes de la sociedad. El profesor ha de ser un excelente comunicador, "es imposible no comunicar, todo lo comunica y toda la comunicación tiene múltiples significados..." (Lozano, 2011:19. El profesor tiene como reto de ser actor, que brote de la creatividad e ingenio, "la actuación, la indumentaria de un personaje y la ambientación del escenario son recursos imprescindibles que están a la disposición de cualquier profesor" (Lozano, 2011:19)

El profesor de básica primaria ha de ser asesor, facilitador del aprendizaje pasando de lo particular a lo general. El compromiso es grande, se requiere de docentes que sigan el método de la mayéutica y perfeccionen el arte de hacer preguntas en pro de buscar respuestas, que despierten el pensamiento crítico y promuevan el aprendizaje significativo.

Es necesario comprender qué;

"el profesor como trabajador conforma su identidad profesional por medio de dos conjuntos de condicionantes; de un lado sus propias actitudes, posiciones, modos de hacer, inseguridades, estado psicológico, trayectoria personal, costes en energía, etc.; y por otro lado las ideas, patrones de acción imaginarios profesionales pedagógicos, modos de pensar, hacer y sentir la educación..." (Prendes; 2010)

Se ha de transformar la educación pensando en la profesionalización del profesor a fin en la disciplina de su saber e integrarlo con su quehacer y ofrecerle la motivación como docente investigador, promover entornos de aprendizaje para los docentes y recomposición de los entornos de trabajo al igual que una mejor remuneración económica y una acogía agradable en su trabajo a fin de una mejor calidad de vida: El profesor de básica primaria ha de ser feliz con y en lo que hace, dimensión vital de su ejercicio profesional en cada comunidad, con cada grado y en la enseñanza de los contenidos de las áreas; en fin un persona integral, idónea $y$ feliz.

Según Tébar (2003), manifiesta una consideración importante y es la del profesor como un mediador de los aprendizajes de los estudiantes y que se aplica para Educación Básica Primaria, cuyos rasgos fundamentales los clasifica en el siguiente orden: 
- Es un experto que domina los contenidos, planifica y es flexible

- Establece metas: perseverancia, hábitos de estudio, autoestima, meta cognición...; siendo su principal objetivo que el mediado construya habilidades para lograr su plena autonomía.

- Regula los aprendizajes, favorece y evalúa los progresos; su tarea principal es organizar el contexto en el que se ha de desarrollar el sujeto, facilitando su interacción con los materiales y el trabajo colaborativo.

- Fomenta el logro de aprendizajes significativos, transferibles...

- Fomenta la búsqueda de la novedad: curiosidad intelectual, originalidad, pensamiento convergente.

- Potencia el sentimiento de capacidad: autoimagen, interés por alcanzar nuevas metas...

- Enseña qué hacer, cómo, cuándo y por qué, ayuda a controlar la impulsividad

- Comparte las experiencias de aprendizaje con los alumnos: discusión reflexiva, fomento de la empatía del grupo...

- Atiende las diferencias individuales

- Desarrolla en los alumnos actitudes positivas: valores...

El profesor como mediador del proceso de enseñanza y aprendizaje para la vida, cuyo papel de formador no es tanto "enseñar" (explicar-examinar) unos conocimientos que tendrán una vigencia limitada y estarán siempre accesibles, como ayudar a los estudiantes a "aprender a aprender" de manera autónoma en esta cultura del cambio y promover su desarrollo cognitivo y personal mediante actividades críticas y aplicativas que, aprovechando la inmensa información disponible y las potentes herramientas Tecnologías de la Información y la Comunicación, tengan en cuenta sus características formación centrada en el estudiante y les exijan un procesamiento activo e interdisciplinario de la información para que construyan su propio conocimiento y no se limiten a realizar una simple recepción pasivamemorización de la información, aspectos válidos y necesarios desde la educación primaria para asegurar y garantizar el éxito de la formación de los cimientos de la condición humana y de los nuevos conocimientos que se adquieren y asimilan para la consolidación de aprendizaje para la vida.

Competencias de un profesor de básica primaria. En el desarrollo de la investigación se ha mantenido la denominación de profesor y no la de: docente, educador, maestro, instructor o tutor por las connotadas diferencias, pero reconociendo las semejanzas; análisis de que no es objeto de la presente disertación. Se considera pertinente acudir a una definición de profesor como: "la persona que enseña alguna ciencia o arte; es el responsable de orientar y coordinar el proceso de enseñanza - aprendizaje en el aula como en la sala de computo. Asesora al estudiante en el desarrollo de las prácticas y ejercicios que se plantean en el material de estudio..." (De la Torre, 2009).

El docente es aquella persona que contribuye al diálogo entre el estudiante y la cultura, además que su función central "consiste en orientar y guiar la actividad mental constructivista de sus alumnos, a quienes proporciona una ayuda pedagógica ajustada a su competencia; en otras palabras, promoverá el aprendizaje significativo de sus estudiantes" (De la Torre, 2009). Al docente que orienta en educación básica primaria, además de apropiarse de las dimensiones que propone Lozano (2011) figura $\mathrm{N}^{\circ} 1$, le es pertinente desarrollar cinco competencias o atributos que según, De la Torre (2009) se pueden constituir en pilares 
fundamentales en el ejercicio de su labor pedagógica con calidad y eficiencia.

El profesor de básica primaria ha de ser un "experto en la materia que ha de enseñar, así mismo conoce y cuestiona el pensamiento espontáneo y adquiere conocimientos relacionados con el aprendizaje de las ciencias" (De la Torre, 2009: 25). En lo didáctico se refiere a la aptitud o suficiencia para transmitir conocimientos; el docente ha de dominar las técnicas y métodos de enseñanza habitual, despertar la innovación e interacción con el estudiante, elementos primordiales al igual que un adecuado equilibrio psicológico. Su competencia psicológica ha de servir para captar los sentimientos de los demás y saberlos tratar como personas, en beneficio de la construcción de un tejido social proactivo.

El docente que enseña en educación básica primaria, ha de propender por la construcción de competencias que le faciliten la interacción con el estudiante, el buen funcionamiento de las áreas obligatorias $\mathrm{y}$ fundamentales $\mathrm{y}$ el éxito del proceso de enseñanza y aprendizaje, con el apoyo de las herramientas, estrategias didácticas a la vanguardia de los tiempos.

Vale la pena señalar el informe de la OCDE (2008), quien proporciona un discernimiento en relación con las condiciones de trabajo de los maestros y las prácticas de la enseñanza y el aprendizaje en las escuelas. Es importante considerar que el estudio analizó el rol del maestro en la calidad de la educación, dentro de los resultados más importantes del estudio se encuentran:

Los maestros que reciben más desarrollo profesional, creen que trabajan en forma más efectiva.

Los maestros que tienen creencias más fuertes acerca de los métodos de enseñanza, informan que tienen un comportamiento más colaborativo con sus colegas, relaciones más positivas entre maestros y alumnos, y sienten que son más efectivos.

Los maestros que recibe reconocimiento por su buen desempeño por parte el director o de sus colegas, sienten que son más efectivos.

El impacto de liderazgo escolar en el aprendizaje es indirecto, y se mitiga a través de las acciones de los maestros

En la tesis doctoral de Flores (2008), se describe "las percepciones que poseen respecto de sí mismos los profesores del primer y segundo ciclo de Educación Básica de las competencias que movilizan en los procesos de: preparación para la enseñanza, la creación de un ambiente propicio para el aprendizaje de los estudiantes, la enseñanza para el aprendizaje de todos los estudiantes y en sus responsabilidades profesionales" (Montes, A; Gamboa, A; Lago, C. 2013), y de cómo son evaluados por sus superiores jerárquicos los profesores de Educación Básica en esas mismas competencias.

Una educación básica primaria de calidad, entonces, a la luz de Álvarez y Topete (2000) está

"orientada hacia la satisfacción de las necesidades básicas de aprendizaje, el establecimiento de las bases necesarias para aprender a aprender, para la educación permanente; la formación de actitudes y hábitos necesarios para la educación permanente; la formación de actitudes y hábitos necesarios para alcanzar niveles satisfactorios de calidad en la vida humana; para participar con conocimiento de causa en las decisiones de la vida cívica, social, cultural, política y para proponer el desarrollo 
de la renovación de los valores de la cultura humana, en sus dimensiones local, regional, nacional y universal

El profesor de Educación Básica Primara ha de ser competente en los modelos pedagógicos tradicionales y contemporáneos para dinamizar los principios de la pedagogía, la evaluación, la didáctica y la innovación de los saberes de las disciplinas fundamentales de la Educación Básica Primaria en los contextos rural y urbano del sector público y privado; teniendo en cuenta las particularidades de cada escenario y las condiciones de cada estudiante para lograr un impacto que transcienda en la vida de cada comunidad educativa y deje una huella en la historia personal y comunitaria.

\section{A MANERA DE CONCLUSIONES}

El rol del profesor de educación básica primaria, sin duda alguna esta mediado por la normatividad vigente en el sistema educativo colombiano. El punto de partida que a normatividad se refiere parte de la Constitución de 1991, se evidencia la educación como un derecho fundamental, acto seguido se evidencia la Ley 30 de 1992, posteriormente la Ley 115 de 1994 y desde luego todos los decretos reglamentarios en relación a la organización del sistema educativo colombiano.

Las reformas educativas de las últimas tres décadas han contribuido al definir el rol del profesor de educación básica primaria en Colombia, organizando y reorganizando las instituciones formadoras: Escuelas Normales Superiores y Universidades; de educadores en los diferentes niveles de educación incluyendo al que refiere y es objeto de estudio; con el propósito del mejoramiento continuo y la calidad de los educadores. La acreditación de las Escuelas Normales Superiores en las diferentes etapas y en las universidades de los programas de las licenciaturas incluida la de educación básica primaria se ha constituido en un objetivo vital y esencial del Ministerio de Educación Nacional

En necesario aclarar que el rol del profesor de Educación Básica Primaria transciende las barreras, los limites y las fronteras de la normatividad siendo esta apenas una forma de estandarizar la formación, profesionalización, capacitación, vinculación de los profesores; siendo visible su auténtico rol en cada escenario de la geografía colombiana que es diferente como lo es cada ser humano.

\section{REFERENCIAS BIBLIOGRÀFICAS}

Álvarez, Isaías \& Topete, Carlos. (2004). Búsqueda de la calidad de la educación Básica, Conceptos básicos, criterios de evaluación y estrategias de gestión. En: Revista Latinoamericana de estudios educativos. México: Centro de Estudios Educativos. vol. XXXIV, No 003; pp. 11-36.

Ayllon, J. R. (2006). 10 Claves de la educación $1^{\text {a }}$ Ed. México. E. Trillas.

Bohórquez, J. (2017) Requisitos para ser docente. Recuperado en: http:// www.mineducacion.gov.co/1621/ articles-163711_archivo_pdf.pdf

Constitución Política De Colombia (2008). $1^{\text {a }}$ ed. Edición actualizada. Bogotá D. C. Colombia: Ediciones Momo.

Cifuentes, J. y Camargo A. (2015). El perfil del docente universitario en la modalidad a distancia. Revista Citas: Ciencias Innovación Tecnología Ambiente Sociedad, Vol. $1 \mathrm{~N}^{\circ}$ 2. Julio-diciembre 2015 con ISSN 2422-4529

Decreto 1860 (1994) Pedagogía y organización del servicio educativo. $1^{\text {a }}$ ed. Bogotá D. C. Colombia: Editorial Unión. 
Decreto 24502015 por el cual se reglamentan las condiciones de calidad para el otorgamiento y renovación del registro calificado de los programas académicos de licenciatura y los enfocados a la educación

Decreto 1075 de 2015, Único Reglamentario del Sector Educación.

Duque, L. J. (2001). El arte de ser Maestro. $2^{\mathrm{a}}$ Ed. Bogotá. Panamericana S. A.

Duque, L. J. (2001). La misión de educar. $2^{\mathrm{a}} \mathrm{Ed}$ Bogotá. Panamericana S. A.

Resolución 18583 del 2017 Por la cual se establecen las características específicas de calidad de los programas de Licenciatura para la obtención, renovación o modificación del registro calificado.

Ferro, J. Prieto C. \& Quijano L. (2009). Economía, globalización y educación. Revista Educación y Ciencia, Julio-diciembre, Núm. 12. Centro de Investigación y Extensión de las Facultad de Educación, Universidad Pedagógica y Tecnológica de Colombia. Colombia.

Flórez, F. (2008). Las competencias que los profesores de educación básica movilizan en su desempeño profesional docente. Tesis Doctoral. Universidad Complutense De Madrid. España.

Ley 30 de 30 de diciembre de 1992. Por la cual se organiza el servicio público de la Educación Superior. $1^{\text {a }}$ ed. Bogotá D. C. Colombia: Ediciones Momo.

Lozano, R. A. Coordinador (2005). El éxito en la enseñanza: Aspectos didácticos de las facetas del profesor $1^{\text {a }}$ ed. México: Editorial Trillas

Ley 115, Ley General de Educación (2008). $1^{\text {a }}$ ed. Edición actualizada. Bogotá D. Colombia: Ediciones Momo.

Ministerio de Educación Nacional (s.f.). Escuelas Normales Superiores Acreditadas. Recuperado el 03 de enero de 2017, en: http://www.mineducacion.gov.co/1621/ articles208800_archivo_pdf_ens_ acreditadas_junio2_2011.pdf

Ministerio de Educación Nacional (s.f.). Escuelas Normales Superiores en Colombia. Recuperado el 03 de enero de 2017, en: http://www.mineducacion.gov.co/1759/ w3-article-345504.html

Morales, G. et al (1999). Se tú mismo, aprende a ser persona. Trabajo de grado para la especialización en docencia universitaria, Bogotá: Universidad Militar Nueva Granada.

Montes, A; Gamboa, A; Lago, C. (2013). La educación básica en Colombia: Una mirada a las políticas educativas. Revista Saber, Ciencia y Libertad ISSN: 17947154 Vol. 8, No.2. En: https://revistas. unilibre.edu.co/index.php/saber/issue/ view/150

OCDE. Metas educativas 2021. (2008). La educación que queremos para la generación de los Bicentenarios. España: Organización de Estados Iberoamericanos para la Educación, la Ciencia y la Cultura.

Tedesco, J. (2000). Los desafíos de las reformas educativas en América Latina. Revista Pedagogía y Saberes, $N^{\circ}$ 14. Facultad de Educación. Universidad Pedagógica Nacional. Págs. 5-14

Tébar, L. (2003) El perfil del profesor mediador. Madrid: Aula XXI/Santillana

Suárez, D. R. (2010). La educación. Estrategias de enseñanza-aprendizaje, teorías educativas. $2^{\mathrm{a}}$ ed. México: Editorial Trillas

Savater, Fernando (2010). El valor de educar. $1^{\text {a }}$ Ed. México. Editorial Trillas. 